\title{
Analysis and quantification of in vitro myoblast fusion using the LADD Multiple Stain
}

\author{
Rhys McColl, Mthokozisi Nkosi, Celia Snyman, and Carola Niesler \\ Department of Biochemistry, School of Life Sciences, University of \\ KwaZulu-Natal, South Africa
}

BioTechniques 61:323-326 (December 2016) doi 10.2144/000114485

Keywords: fusion; myoblast; microscopy; LADD; skeletal muscle; differentiation

Supplementary material for this article is available at www.BioTechniques.com/article/114485.

Myoblast fusion, which is essential for muscle development, regeneration, and repair, can be assessed in vitro via the calculation of a fusion index. Traditionally, this requires use of either immunocytochemistry or fluorescently-labeled cytoskeletal staining, followed by microscopy and laborious analysis. The expense and time-consuming nature of the optimization and application of antibody-based techniques such as immunocytochemistry, as well as the need for specialized analytical equipment such as fluorescence microscopes, presents a barrier to the routine analysis of this crucial step during terminal differentiation. Here, we describe (I) a novel use of the commonly available LADD Multiple Stain for visualization of myoblast fusion in vitro; (ii) the optimization of a simple image analysis method to generate quick, quantifiable data representative of a fusion index; and (iii) the use of a protocol combining these two procedures to investigate in vitro myoblast fusion in a simple and efficient manner as proof-of-concept.

Adult skeletal muscle tissue contains a population of precursor cells, known as satellite cells, that are responsible for skeletal muscle repair (1-3). Activated satellite cells (myoblasts) migrate to the site of muscle trauma, where they proliferate, differentiate, and fuse into multinucleated myotubes (3-5). The ultimate success of this process is measured by the degree to which resident myoblasts have fused during terminal differentiation.

In order to experimentally evaluate myoblast fusion in vitro, a fusion index is calculated based on detection of immunofluorescence by microscopy.
Fluorescently labeled antibodies are used to detect muscle fiber structural proteins such as myosin heavy chain (MyHC) and desmin, or alternatively, fluorescently labeled phalloidin can be used to visualize F-actin (6-9). Nuclei are fluorescently labeled using a DNA binding compound such as Hoechst 33258. Subsequently, either the percentage of nuclei in myocytes with $\geq 3$ nuclei (9) or the percentage of nuclei in MyHC-positive myotubes (10) is calculated. These approaches are well established; however, they require extensive, time-consuming optimization to generate a strong and specific signal for the antigen of interest and subsequent fusion analysis. If fluorescently labeled antibodies are utilized, access to a fluorescence microscope is an additional requirement not available in all institutions. Subsequent quantitation requires laborious and time-consuming analysis of numerous fields of view in order to obtain a fusion index representative of the population. These limitations, together with the relative expense of antibody-based assays, prompted us to develop a rapid, cost-effective in vitro assay for quantification of myoblast fusion using the widely available LADD Multiple Stain.

The LADD Multiple Stain (11) is a combined nuclear and cytoplasmic stain that contains fuchsin and toluidine blue (Cat. \#70955; LADD Research Industries, Williston, VT). For our studies, fresh LADD is prepared to contain 0.365 g toluidine blue (Cat. \#89640-5G; Sigma-Aldrich, St. Louis, MO) and 0.135 g fuchsin (Cat. \#47860-25G; SigmaAldrich) in a final volume of $50 \mathrm{ml}$ of $30 \%$ ethanol. The solution is mixed until dissolved and then filtered through Whatman (number 4) filter paper (Cat. \#09-825B, Thermo Fisher Scientific, Waltham, MA). LADD stain can be stored in a plastic or glass container at room temperature and can be reused. Cells to be stained are washed with phosphatebuffered saline (PBS) and fixed in 70\% ethanol for $10 \mathrm{~min}$. The ethanol is removed, and $500 \mu \mathrm{L}$ LADD stain is added, ensuring full coverage of cells. Cells are incubated for $1 \mathrm{~min}$, after which the stain is removed, and the cells are repeatedly washed with distilled water until the LADD ceases to leach into the water. The cells are then left to dry and stored at room temperature until viewed using phase-contrast light microscopy. To improve lighting during viewing, the stained cells can be immersed in PBS.

To determine whether this stain can be successfully utilized to visualize myoblast fusion, C2C12 cells were grown to $80 \%$ confluence (Figure $1 \mathrm{~A}$ ) under standard culture conditions (12). The media was then changed to differentiation media containing $2 \%$ horse

\section{METHOD SUMMARY}

The use of the LADD Multiple Stain and automated ImageJ analysis provides a rapid, cost-effective method for analyzing and quantifying myoblast fusion. 
serum, resulting in fusion into myotubes (Figure 1B). Successful differentiation was confirmed by increased expression of $\mathrm{MyHC}$ (Figure 1D) compared with undifferentiated cells (Figure 1C). Following LADD staining, the undifferentiated C2C12 myoblasts display a light purple cytoplasm and a darker nucleus (Figure 1E; arrow). However, following differentiation, myotube cytoplasm appears dark purple (Figure 1F; arrowheads), while lighter nuclei are clearly visible within the multinucleated cell (Figure 1F; arrow). Therefore, following LADD Multiple Stain, clearly defined nuclei are observed and are as easily distinguishable from the cytoplasm of the multinucleated myotube (Figure 1F) as viewed using fluorescence microscopy following immunocytochemistry (Figure 1D).

To determine whether image analysis could be used to measure the progress of myoblast fusion, C2C12 cells were differentiated for 6 days, and images of LADDstained cells taken at $200 \times$ magnification using an Olympus CKX41 inverted light microscope (Olympus Corporation, Tokyo, Japan). As expected, the number of fusing myocytes and myotubes clearly increased at each subsequent differentiation day (Figure 2A). A fusion index was then calculated, and differentiating myoblasts were seen to incrementally increase their fusion index from $0.45 \%$ (Day 1) to 31.4\% (Day 6) (Figure 2B). The fusion index derived in this way compares favorably with the index calculated using standard immunocytochemistry (to detect $\mathrm{MyHC}$ ) and nuclear staining (13). To determine whether ImageJ analysis software (https://imagej.nih.gov/ij/) could be adapted to quantify myofiber area (as a measure of fusion), a macro was developed and tested on the images represented by Figure 2A. The macro is set up as follows and then saved:

1. Open ImageJ and click "Plugins $=>$ Macros = > Startup Macros"

2. Replace the existing text with the coding shown in Supplementary Table S1.

The images are opened in ImageJ, and the macro is run by clicking "Macros $=>$ Run Macro". This will analyze the pictures one at a time. The user must confirm that the threshold has been set correctly; only the myofiber area should be analyzed. The color threshold can

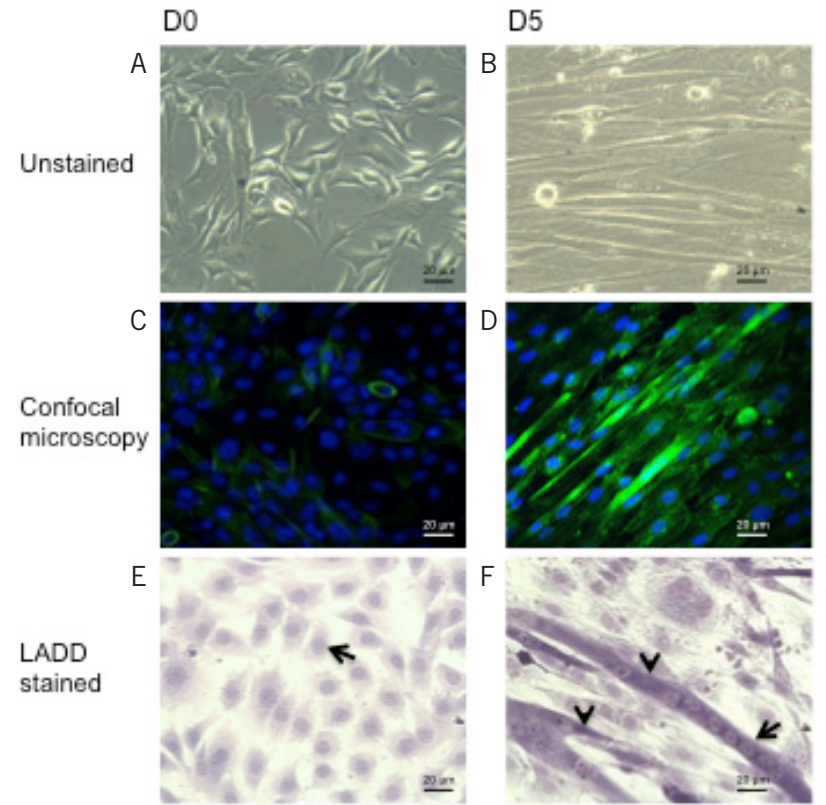

Figure 1. Visualization of multinucleated myofibers. $\mathrm{C} 2 \mathrm{C} 12$ myoblasts were cultured in differentiation media for 5 days. Cells were evaluated at Day 0 (D0) and Day 5 (D5) of differentiation. Phase contrast light microscopy images of unstained myoblasts (A) at D0 and myotubes (B) at D5. Confocal microscopy images of myoblasts (C) at D0 and myotubes (D) at D5 labeled for myosin heavy chain (MyHC) (green) and co-stained with Hoechst 33258 to visualize nuclei (blue). MyHC was detected using the mouse monoclonal MF20 primary antibody (1:200 dilution) (Developmental Studies Hybridoma Bank) followed by DyLight 488-conjugated AffiniPure donkey anti-mouse IgG secondary antibody (Jackson ImmunoResearch, West Grove, PA). Phase contrast microscopy images of LADD-stained myoblasts (E) at DO and myotubes (F) at D5; arrows indicate nuclei; arrowheads indicate cytoplasmic staining. Scale bars $=20 \mu \mathrm{m}$.
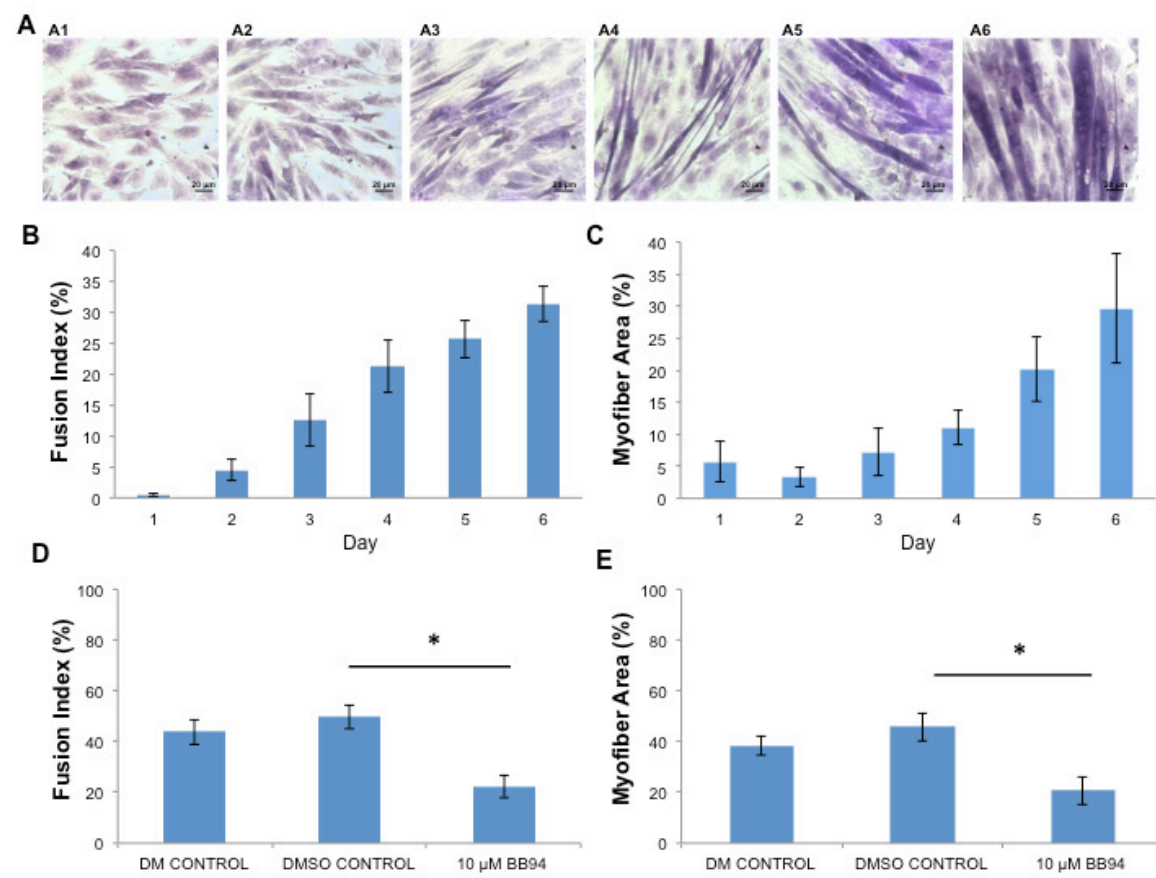

E



Figure 2. Analysis of differentiation using LADD Multiple Stain. C2C12 myoblasts were differentiated and stained with LADD. (A) LADD-stained images of cells taken at days 1-6 of differentiation (A1A6). (B) Percentage fusion, calculated at each day of differentiation by dividing the number of nuclei within multinucleated myofibers by the total number of nuclei. (C) Assessment of myofiber area, at each day of differentiation, following automated analysis of images using the ImageJ macro and analysis software. (D) Fusion index of differentiating cells (Day 5) cultured in the presence or absence of BB94 $(10 \mu \mathrm{M})$. (E) Myofiber area of differentiating cells (Day 5) cultured in the presence or absence of BB94 $(10 \mu \mathrm{M})$. Data are expression as mean \pm SEM; $n=4 ;{ }^{*} P<0.05$. Scale bars $=20 \mu \mathrm{m}$. 
be changed by editing the macro line "setThreshold $(0,130)$ "; by increasing the second number, more lightly stained areas will be included, whereas more areas are excluded when this number is reduced. Using this method, myofiber area reached 29.6\% at Day 6 (Figure $2 \mathrm{C})$, comparing well with the fusion index calculated for the same time point (Figure 2B). Jenner and Giemsa dyes can also be used to stain myotubes for light microscopy in much the same way as LADD, however staining with LADD is many times faster, and the ImageJ macro we developed allows for greater control over which areas are measured (14).

To further validate the use of the LADD Multiple Stain for fusion analysis, C2C12 cells were differentiated for 5 days in the presence or absence of $10 \mu \mathrm{M}$ BB94 (Cat. \#ab142087; Abcam, Cambridge, UK) and subsequently fixed and stained with LADD as described earlier. BB94 is a commercially available synthetic matrix metalloprotease inhibitor that has previ- ously been shown to reduce myoblast fusion $(15,16)$. In the presence of BB94, the fusion index decreased significantly from 50\% (DMSO control) to $22 \%(P<0.05)$ (Figure 2D); analysis of the myofiber area revealed the same trend, with fusion reduced from $46 \%$ (DMSO control) to $21 \%(P<0.05)$ in the presence of BB94 (Figure 2E).

Here, we present a rapid, novel method for staining both myofiber structure and associated myonuclei using the LADD Multiple Stain. ImageJ is subsequently used to accurately evaluate muscle fiber area as a measure of fusion. The relatively low cost of LADD and the greatly reduced time required for processing and analysis means that fusion can now rapidly be analyzed in a standard laboratory without the need for immunocytochemistry and fluorescence microscopy.

\section{Author contributions}

R.M. and M.N. were responsible for the planning and execution of the laboratory work, as well as compilation and revision of the manuscript. C.S. provided intellectual input and supervision to the students involved, and also contributed to revisions of multiple drafts of the article. C.N. provided intellectual input and supervision to the students and postdoctoral scientist involved, provided the funding for the project, and contributed to revisions of multiple drafts of the article.

\section{Acknowledgments}

The work was supported by the South African National Research Foundation, South African Medical Research Council and University of KwaZulu-Natal. The authors also thank the UKZN Microscopy and Microanalysis Unit (Pietermaritzburg) for all their assistance. The MF20 monoclonal antibody, developed by Donald, A. Fischman, M.D., was obtained from the Developmental Studies Hybridoma Bank developed under the auspices of the National Institute of Child Health and Human Development (NICHD) and maintained by the University of lowa, Department of Biology, lowa City, IA.

\section{DETECT CRISPR GENE EDITING EVENTS Using Agarose Gels}

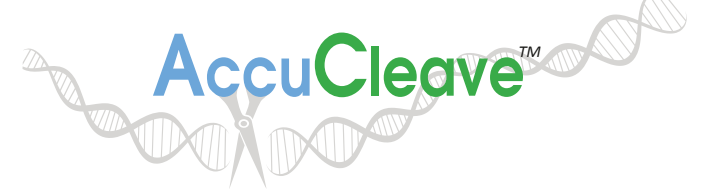

Streamline gene editing workflows with a simple and accurate enzymatic assay.

\section{AccuCleave $^{\mathrm{TM}}$ T7 Kit}

- T7 based enzyme kit for mutation frequency detection of CRISPR events.

\section{AccuCleave $^{\mathrm{TM}}$ C2 Control DNA Kit}

- Contains 2 Control DNA fragments - intact and -2 deletion fragment.

\section{AccuCleave $^{\mathrm{TM}}$ CI5 Control DNA Kit}

- Contains 15 Control DNA fragments - intact, $\pm 1, \pm 2, \pm 10 \mathrm{lnDels}$, and 8 point mutations.

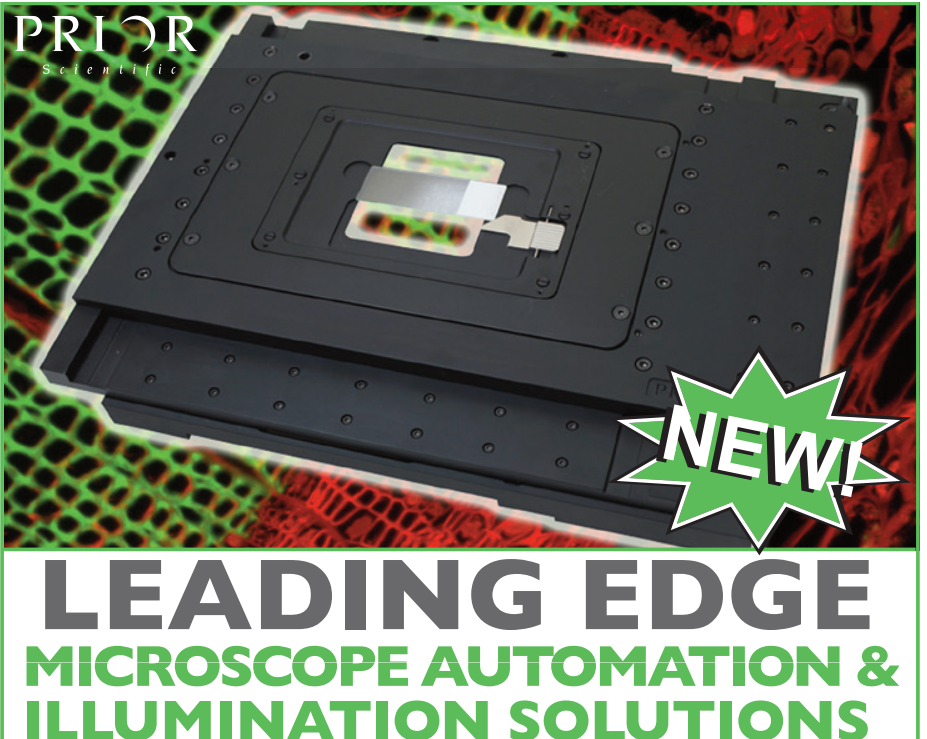

From the highest precision linear motor microscope stages to the most advanced fluorescence illumination systems, Prior Scientific manufactures the highest performing and most reliable microscope automation equipment available.
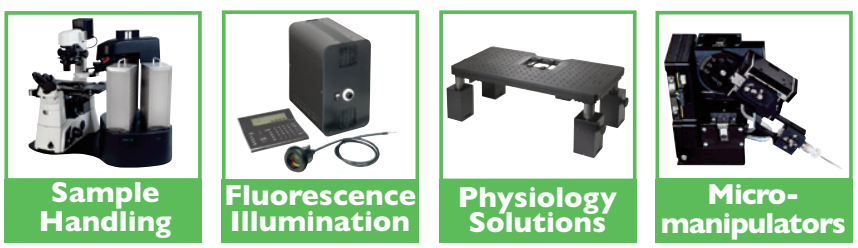

Prior Scientific, Inc. 80 Reservoir Park Dr. Rockland, MA. 02370 Tel: 800-877-2234 Web: www.prior.com 


\section{Competing interests}

The authors declare no competing interests.

\section{References}

1. Hawke, T.J. and D.J. Garry. 2001. Myogenic satellite cells: physiology to molecular biology. J. Appl. Physiol. 91:534-551.

2. Chargé, S.B.P. and M.A. Rudnicki. 2004. Cellular and molecular regulation of muscle regeneration. Physiol. Rev. 84:209-238.

3. Kuang, S., K. Kuroda, F. Le Grand, and M.A. Rudnicki. 2007. Asymmetric self-renewal and commitment of satellite stem cells in muscle. Cell 129:999-1010.

4. Burdzinska, A., K. Gala, and L. Paczek. 2008. Myogenic stem cells. Folia Histochem. Cytobiol. 46:401-412.

5. Abmayr, S.M. and G.K. Pavlath. 2012. Myoblast fusion: lessons from flies and mice. Development 139:641-656.

6. Martin, N.R., S.L. Passey, D.J. Player, A. Khodabukus, R.A. Ferguson, A.P. Sharples, V. Mudera, K. Baar, and M.P. Lewis. 2013. Factors affecting the structure and maturation of human tissue engineered skeletal muscle. Biomaterials 34:5759-5765.

7. Walker, N., T. Kahamba, N. Woudberg, K. Goetsch, and C. Niesler. 2015. Dosedependent modulation of myogenesis by HGF: implications for c-Met expression and downstream signalling pathways. Growth Factors 33:229-241.
8. Makarenkova, H.P., K.N. Gonzales, W.B. Kiosses, and R. Meech. 2009. Barx2 controls myoblast fusion and promotes MyoDmediated activation of smooth muscle-actin gene. J. Biol. Chem. 284:14866-14874.

9. Sharples, A.P., D.J. Player, N.R. Martin, V. Mudera, C.E. Stewart, and M.P. Lewis. 2012. Modelling in vivo skeletal muscle ageing in vitro using three-dimensional bioengineered constructs. Aging Cell 11:986-995.

10. Nishiyama, T., I. Kii, and A. Kudo. 2004. Inactivation of Rho/ROCK signalling is crucial for the nuclear accumulation of FKHR and myoblast fusion. J. Biol. Chem. 279:4731147319.

11. Kaplow, L.S. and C. Ladd. 1965. Brief Report: Simplified Myeloperoxidase Stain Using Benzidine Dihydrochloride. Blood 26:215-219.

12. Burattini, S., P. Ferri, M. Battistelli, R. Curci, F. Luchetti, and E. Falcieri. 2004. C2C12 murine myoblasts as a model skeletal muscle developement: morpho-functional characterization. Eur. J. Histochem. 48:223-233.

13. Micheli, L., L. Leonardi, F. Conti, G. Maresca, S. Colazingari, E. Mattei, S.A. Lira, S. Farioli-Vecchioli, et al. 2011. PC4/ Tis7/IFRD1 stimulates skeletal muscle regeneration and is involved in myoblast differentiation as a regulator of MyoD and NF-kappaB. J. Biol. Chem. 286:5691-5707.

14. Veliça, P. and C. Bunce. 2011. A Quick, Simple And Unbiased Method To Quantify C2C12 Myogenic Differentiation. Muscle Nerve 44:366-370.
15. Botos, I., L. Scapozza, D. Zhang, L.A. Liotta, and E.F. Meyer. 1996. Batimastat, a potent matrix meallo proteinase inhibitor, exhibits an unexpected mode of binding. Proc. Natl. Acad. Sci. USA 93:2749-2754.

16. Ohtake, Y., H. Tojo, and M. Seiki. 2006. Multifunctional roles of MT1-MMP in myofiber formation and morphostatic maintenance of skeletal muscle. J. Cell Sci. 119:3822-3832.

Received 05 August 2016; accepted 08 September 2016

Address correspondence to Carola Ulrike Niesler, Department of Biochemistry, School of Life Sciences, University of KwaZulu-Natal, Private Bag X01, Scottsville 3209, South Africa. E-mail: carolaniesler@gmail.com

To purchase reprints of this article, contact: biotechniques@fosterprinting.com

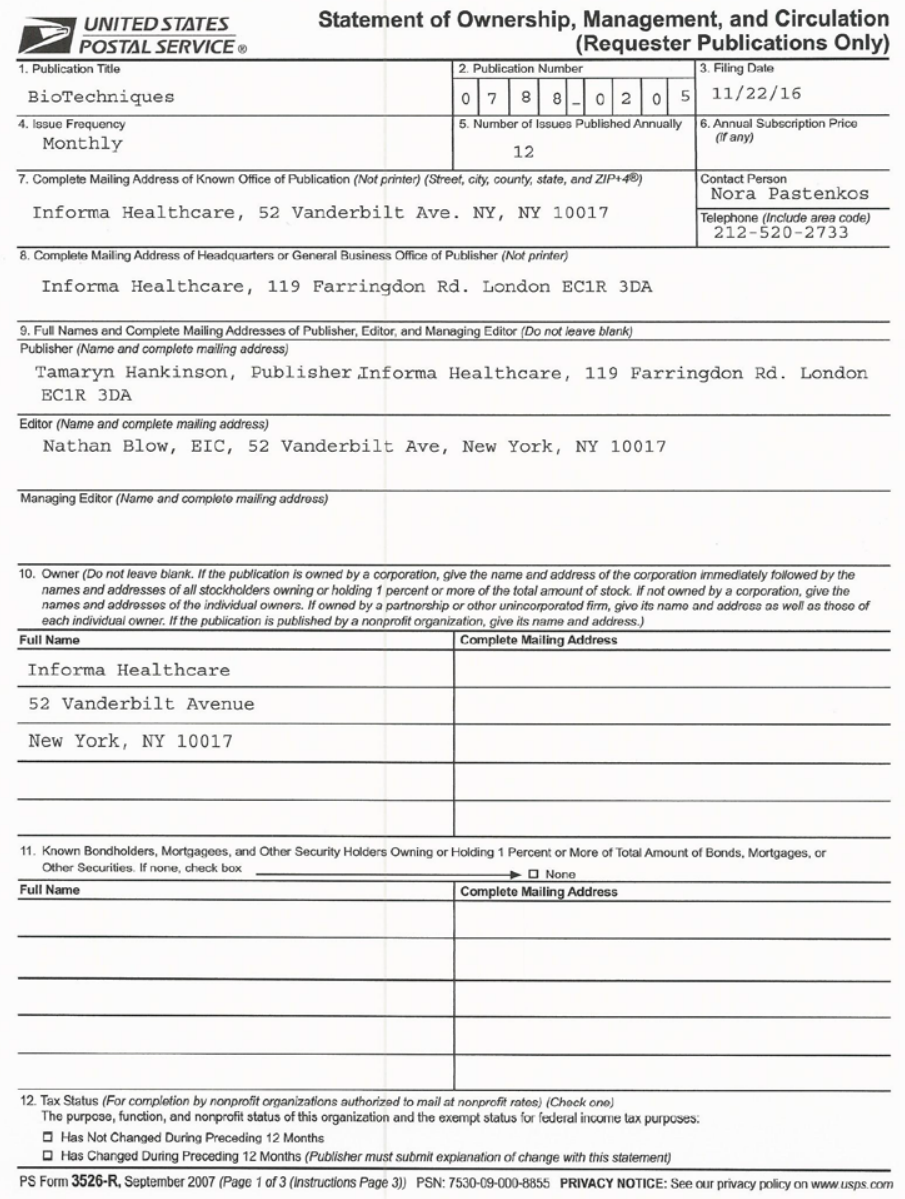

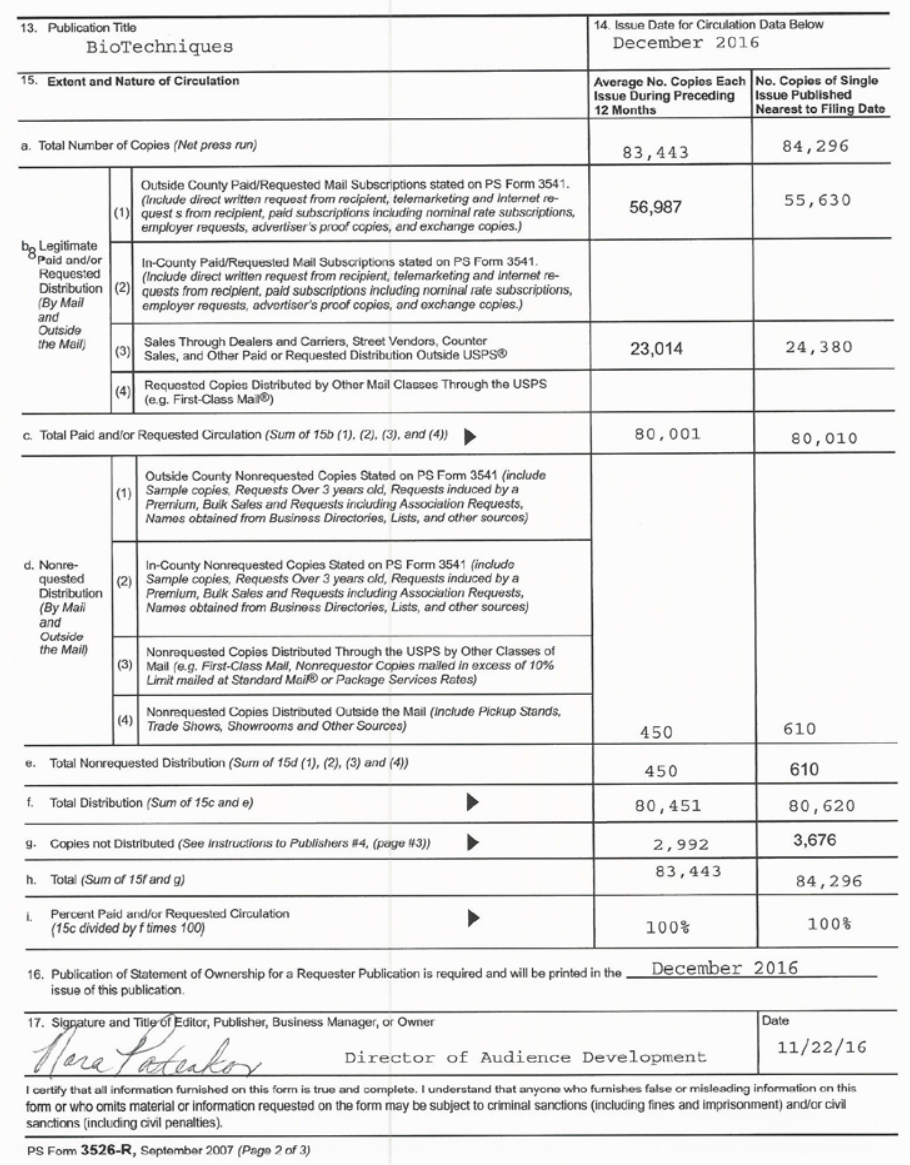

